\title{
OIP5-AS1 Promotes Proliferation of Non-small-cell Lung Cancer and Head and Neck Squamous Cell Carcinoma Cells
}

\author{
YOJIRO KOTAKE $^{1,2}$, NATSUMI MATSUNAGA ${ }^{1}$, TAKAHIRO WAKASAKI ${ }^{3}$ and RYOTARO OKADA ${ }^{1}$ \\ ${ }^{1}$ Graduate School of Humanity-Oriented Science and Engineering, Kindai University, Fukuoka, Japan; \\ ${ }^{2}$ Department of Biological and Environmental Chemistry, \\ Faculty of Humanity-Oriented Science and Engineering, Kindai University, Fukuoka, Japan; \\ ${ }^{3}$ Department of Otorhinolaryngology, Graduate School of Medical Sciences, Kyushu University, Fukuoka, Japan
}

\begin{abstract}
Background/Aim: The long noncoding RNA OIP5 antisense RNA 1 (OIP5-AS1) is overexpressed in various cancer types, such as lung cancer, hepatoblastoma and cervical cancer, and functions to accelerate cell proliferation, invasion and migration. Here, we investigated the roIe of OIP5-AS1 in cell-cycle progression of H1299 and A549 non-small cell lung cancer cells, and FaDu and CAL27 head and neck squamous cell carcinoma cells. Materials and Methods: The cells were transfected with small interfering RNA and subjected to cell-cycle analysis and reverse-transcription quantitative polymerase chain reaction (RT-qPCR). Results: Silencing of OIP5-ASI suppressed the proliferation of H1299, A549, FaDu and CAL27 cells. RT-qPCR and cell-cycle analysis revealed that silencing OIP5-AS1 increased the expression of CDK inhibitors, such as p15, p16, p18 and p19, resulting in G1phase arrest. Conclusion: OIP5-AS1 regulates G1-phase progression by repressing $C D K$ inhibitors and, thus, promotes the proliferation of H1299, A549, FaDu and CAL27 cells.
\end{abstract}

OPA-interacting protein 5 antisense transcript 1 (OIP5-AS1) is a long noncoding RNA (lncRNA) identified as the mammalian homolog of cyrano in zebrafish. In zebrafish, cyrano is essential for embryonic development (1). We

This article is freely accessible online.

Correspondence to: Yojiro Kotake, Ph.D., Department of Biological and Environmental Chemistry, Faculty of Humanity-Oriented Science and Engineering, Kindai University, 11-6 Kayanomori, Iizuka, Fukuoka 820-8555, Japan. Tel: +81 0948225659 (ext. 469), Fax: +81 0948230536, e-mail: ykotake@fuk.kindai.ac.jp

Key Words: Long noncoding RNA, OIP5-AS1, CDK inhibitor, head and neck squamous cell carcinoma, non-small-cell lung cancer. previously showed that silencing human OIP5-AS1 inhibited the proliferation of HeLa cells by causing cell-cycle arrest at the $\mathrm{G}_{2} / \mathrm{M}$ phase, implying that human OIP5-ASI functions to promote cell proliferation (2). Recent studies with clinical cancer specimens clarified that OIP5-ASl is overexpressed in various cancer types such as of the breast, lung, cervix and bladder, as well as glioma, osteosarcoma and hepatoblastoma (3). OIP5-ASI also participates in both positive and negative regulation of cell proliferation in cervical cancer $(2,4)$. Moreover, in many different human cancer cell types, including those of lung cancer (5), osteosarcoma (6), glioma (7) and hepatoblastoma (8), OIP5-AS1 functions to accelerate cell proliferation, invasion, migration and prevent apoptosis, so it is thought to function as an oncogene in these kinds of cancer. OIP5-ASI acts as a sponge for many microRNAs (miRNAs) thereby inhibiting them, resulting in the regulation of WNT/ $\beta$-catenin, PI3K/AKT serine/threonine kinase 1 and NOTCH signalling pathways (9).

Cell proliferation is strictly regulated by the cell cycle. The progression of the cell cycle is accelerated by a series of complexes consisting of cyclins and cyclin-dependent kinases (CDKs) and is repressed by CDK inhibitors (CKIs) (10). CKIs comprise two groups, the INK4 family including p15, p16, p18 and p19 and the Cip/Kip family including p21, p27 and p57 (11). Forced expression of these CKIs results in $\mathrm{G}_{1}$ phase arrest in the cell cycle. Regulation of the $\mathrm{G}_{1}$ phase by cyclin/CDK complexes and CKIs is thought to be important for the regulation of cell proliferation. Recently, we revealed that a lncRNA, antisense non-coding RNA in the INK4 locus (ANRIL), functions as a positive regulator of $\mathrm{G}_{1}$-phase progression in head and neck squamous cell carcinoma cells by repressing p15 and p16 expression (12). However, the involvement of OIP5-AS1 in cell-cycle regulation is largely unknown. In the present work, we studied the role of OIP5-AS1 in cell-cycle regulation in nonsmall-cell lung cancer (NSCLC) and head and neck squamous cell carcinoma cells. 


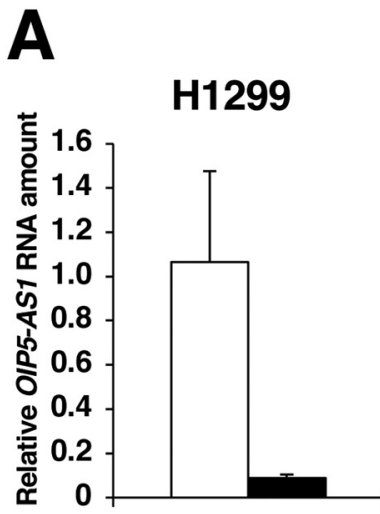

Ctr-i SiRNA

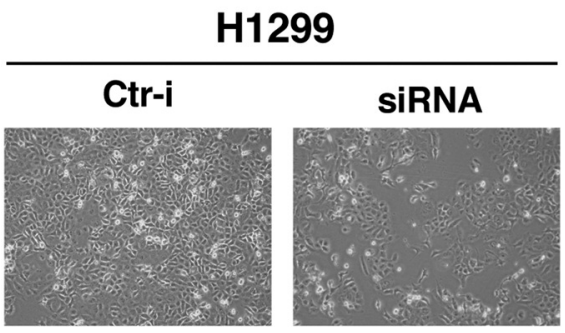

H1299

B

A549
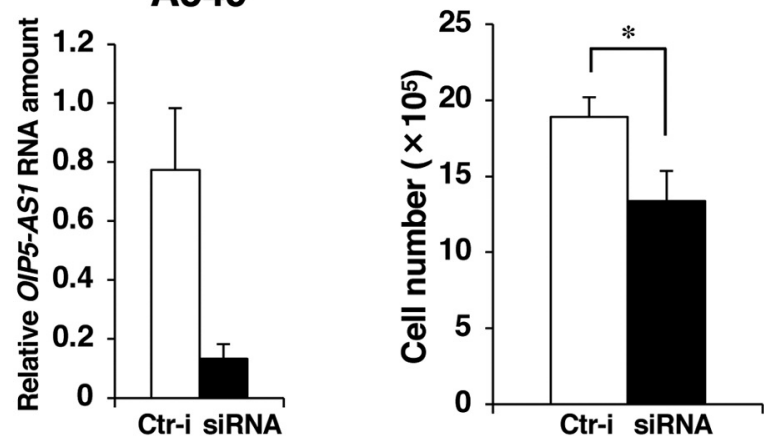

$\mathrm{FaDu}$
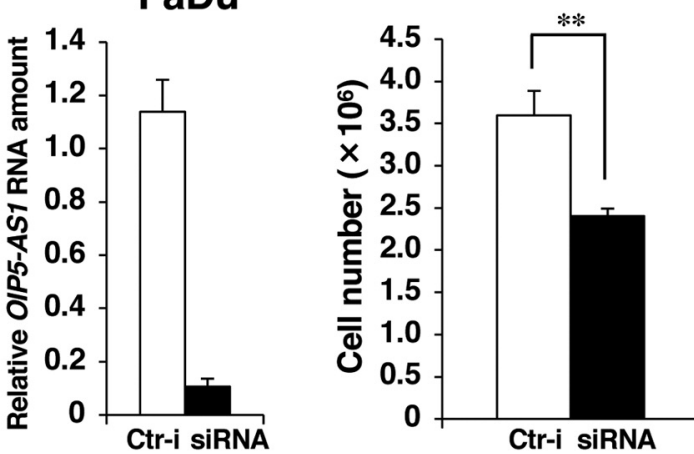

D
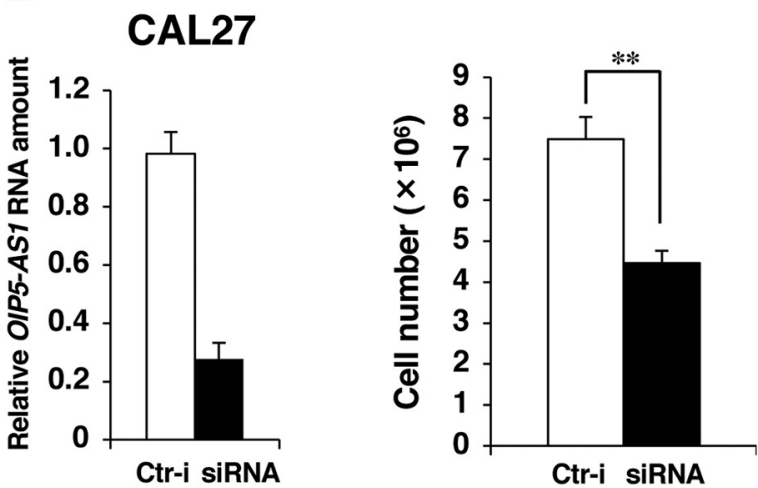

Figure 1. Silencing long noncoding RNA OIP5 antisense RNA 1 (OIP5-AS1) suppresses the proliferation of lung cancer and head and neck squamous cell carcinoma cells. The expression level of OIP5-AS1 was determined by reverse-transcription quantitative polymerase chain reaction (left panels). The data for silenced cells (siRNA) are expressed relative to the corresponding values for cells transfected with control siRNAs (Ctr-i). After transfection with siRNAs for $72 \mathrm{~h}$, the viable cell number was determined by trypan blue staining (right panels). A: H1299 cells. Middle panel shows images under phase-contrast microscopy at $72 \mathrm{~h} ; \mathrm{B}$ : A549 cells; C: FaDu cells; and D: CAL27 cells. Significantly different at: *p<0.05 and **p<0.01.

\section{Materials and Methods}

Cell culture and RNA interference. H1299 and A549 human NSCLC cells, FaDu hypopharyngeal cancer cells, and CAL27 human oral cancer cells were acquired from the American Type Culture
Collection (Frederick, MD, USA). These cells were cultured in Dulbecco's modified Eagle's medium supplemented with $10 \%$ foetal bovine serum (GIBCO, Grand Island, NY, USA). They were seeded at $5 \times 10^{5}$ cells in a $100 \mathrm{~mm}$ dish and cultured for $24 \mathrm{~h}$, after which they were transfected with small interfering RNAs (siRNAs) using 
A

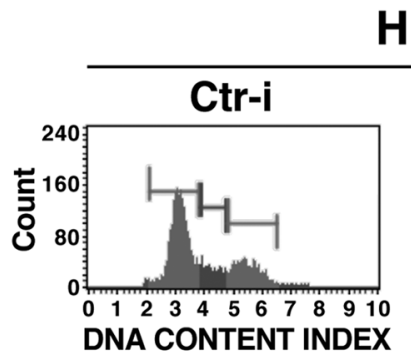

H1299
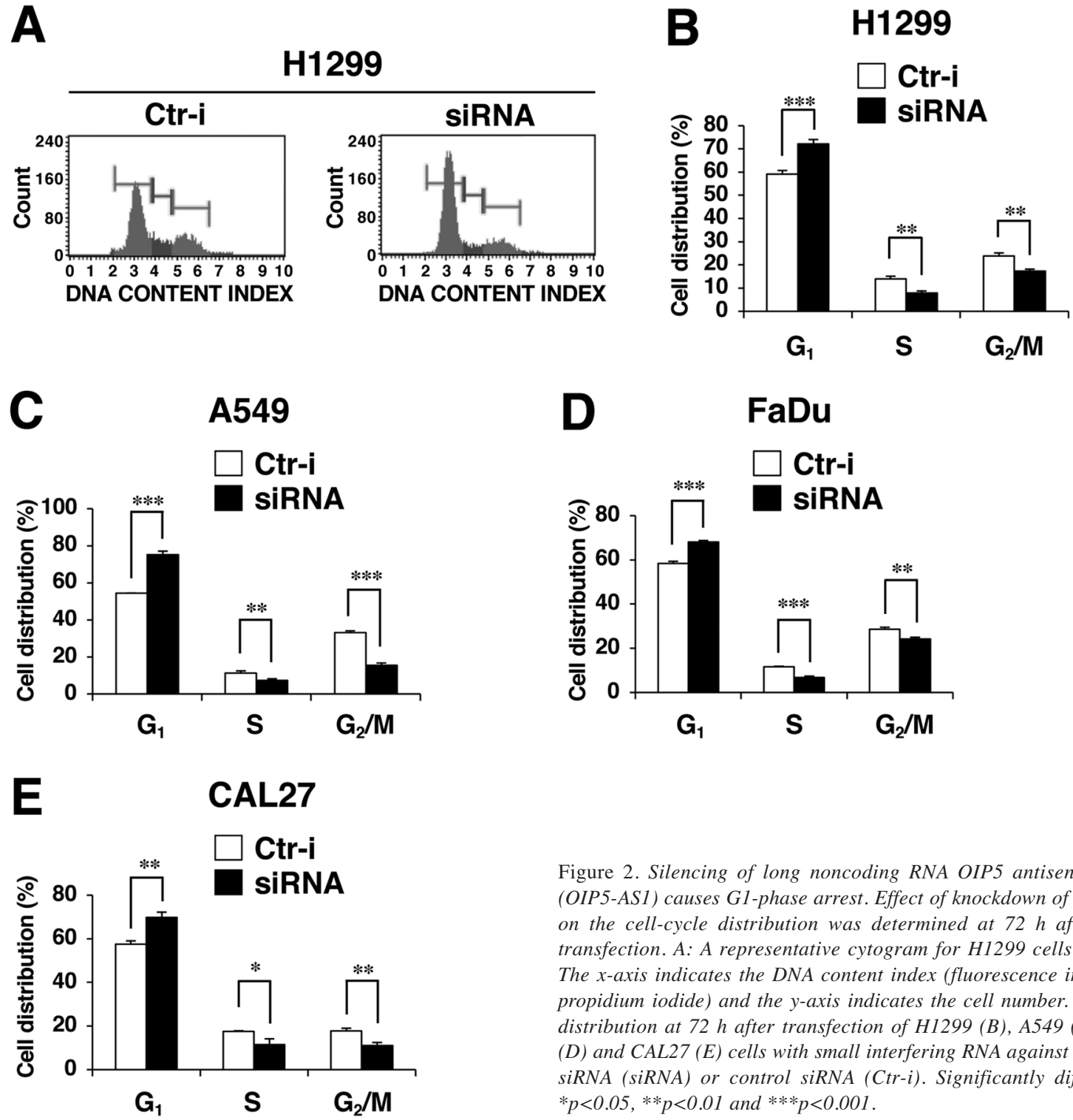

Lipofectamine RNAiMAX (Thermo Fisher Scientific, Waltham, MA, USA). The nucleotide sequence of siRNAs against OIP5-ASI was 5'-GCAGCAUGCUGUGUGCAAA-3' with $3^{\prime}$ ' dTdT overhangs.

The 3D culture of cells was performed as described previously (13). Briefly, 1,000 cells were seeded in a 96-well plate with an ultra low attachment surface and round bottom (Corning, NY, USA) and cultured for $72 \mathrm{~h}$. Images of spheroids were then obtained under a CKX41 inversed microscope (Olympus, Tokyo, Japan). The spheroid areas were measured by Image $\mathbf{J}$ software.

Cell-cycle analysis. The cells transfected with siRNAs were collected at $72 \mathrm{~h}$ after siRNA transfection and fixed with $70 \%$ ethanol. The fixed cells were stained by a Muse ${ }^{\circledR}$ Cell Cycle Kit (Luminex, Toronto, Canada), in accordance with the manufacturer's

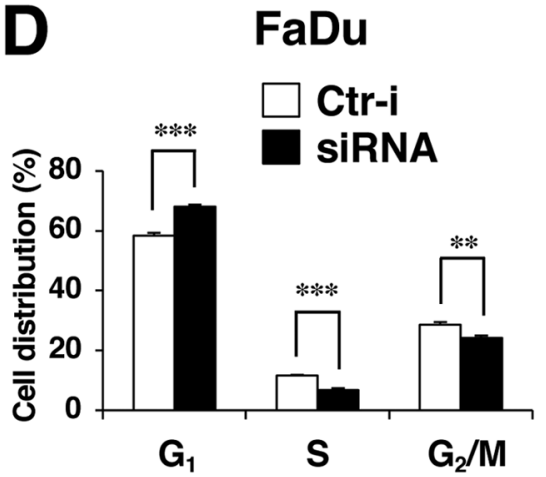

Figure 2. Silencing of long noncoding RNA OIP5 antisense RNA 1 (OIP5-AS1) causes G1-phase arrest. Effect of knockdown of OIP5-AS1 on the cell-cycle distribution was determined at $72 \mathrm{~h}$ after siRNA transfection. A: A representative cytogram for H1299 cells is shown. The $x$-axis indicates the DNA content index (fluorescence intensity of propidium iodide) and the y-axis indicates the cell number. Cell-cycle distribution at $72 \mathrm{~h}$ after transfection of H1299 (B), A549 (C), FaDu (D) and CAL27 (E) cells with small interfering RNA against OIP5-AS1 siRNA (siRNA) or control siRNA (Ctr-i). Significantly different at: $* p<0.05, * * p<0.01$ and $* * * p<0.001$.

protocol and analysed using a Muse ${ }^{\circledR}$ Cell Analyzer (Merck Millipore, Darmstadt, Germany).

Reverse-transcription quantitative polymerase chain reaction (RT$q P C R)$. The cells transfected with siRNAs were collected at $72 \mathrm{~h}$ after siRNA transfection and subjected to RT-qPCR as described previously (14). The nucleotide sequence of the primer set used for OIP5-AS1 was 5'-AAGCTGCCTTGAGAACTCAGAA-3' and 5'-TGTCAGCAAAT GCTGGACTCT-3'. The nucleotide sequences of the primer sets for p15, p16 (15), p18, p19,p21,p27, p57 and glyceraldehyde 3-phosphate dehydrogenase have been described elsewhere (16).

Statistical analysis. The data in graphs are presented as the means and standard derivations of experiments were performed three times. The statistical analysis was carried out by two-tailed $t$-test. A 

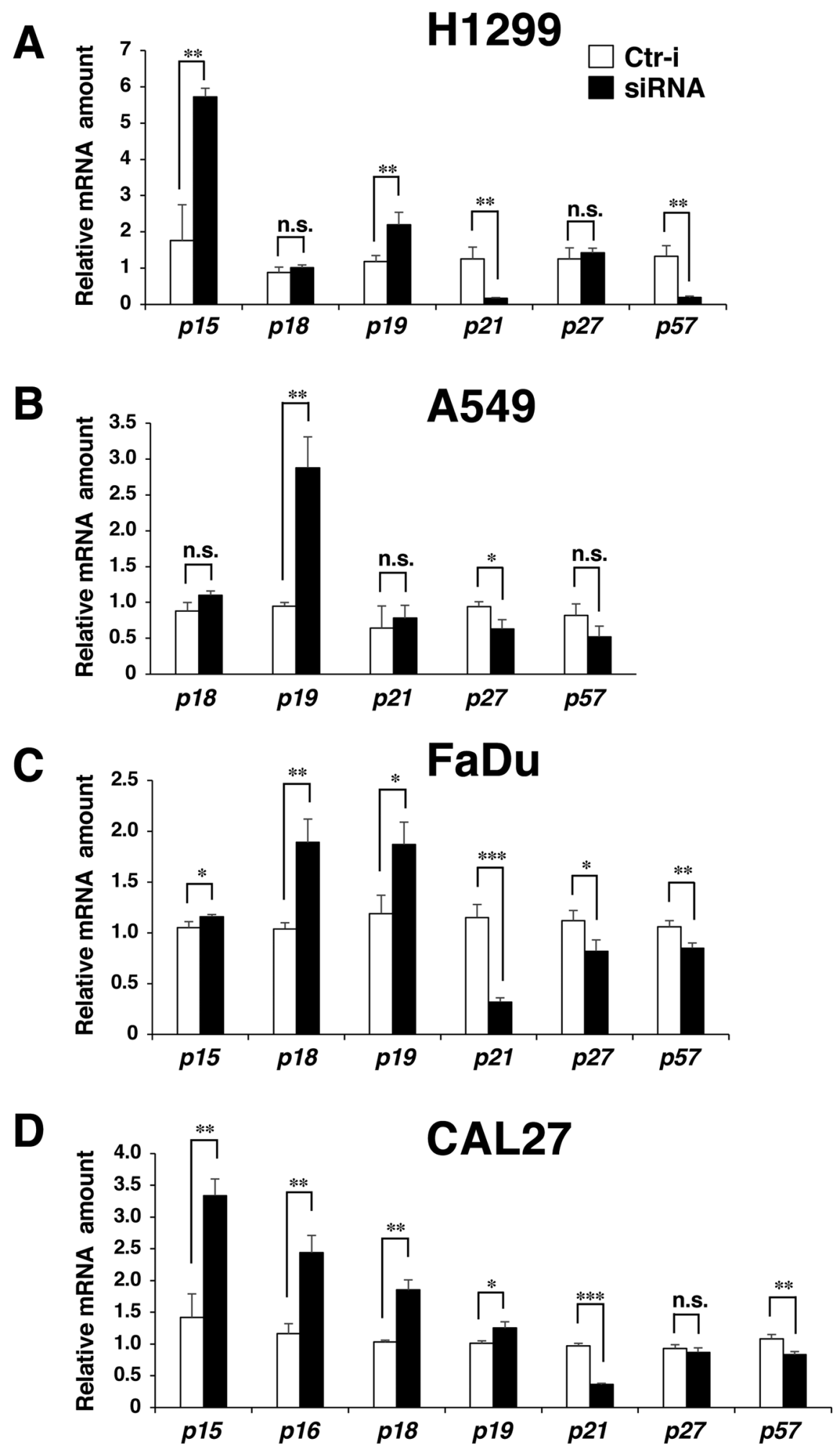

Figure 3. The effect of silencing of long noncoding RNA OIP5 antisense RNA 1 (OIP5-AS1) on expression of cyclin-dependent kinase inhibitors. H1299 (A), A549 (B), FaDu (C) and CAL27 (D) cells were transfected with small interfering RNA against OIP5-AS1 siRNA (siRNA) or control siRNA (Ctr-i), At 72 h after siRNA transfection, the cells were collected. The expression levels of cyclin-dependent kinase inhibitors were measured by reverse-transcription quantitative polymerase chain reaction. The data are expressed relative to the corresponding value for the Ctr-i cells. Significantly different at: $* p<0.05$, **p<0.01 and ***p<0.001; n.s.: not significant. 
A

\section{H1299}

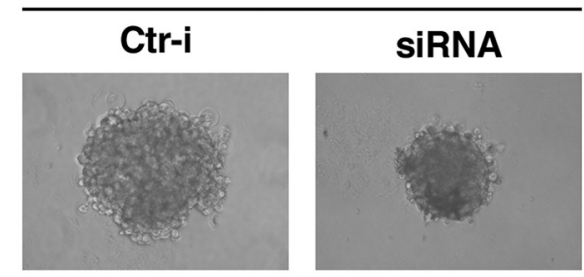

B

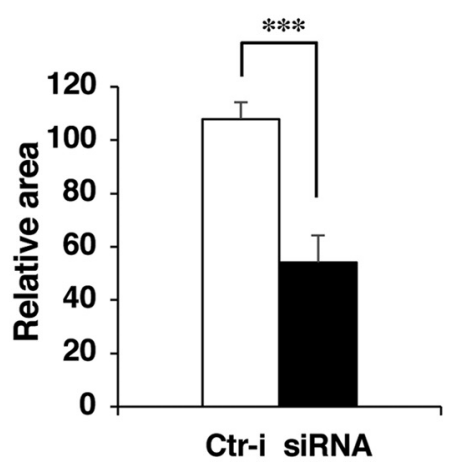

Figure 4. Silencing of long noncoding RNA OIP5 antisense RNA 1 (OIP5-AS1) inhibits the 3D proliferation of $H 1299$ cells. A: Images of the spheroids produced by $H 1299$ cells in $3 D$ culture for $72 \mathrm{~h}$ after transfection with small interfering RNA against OIP5-AS1 siRNA (siRNA) or control siRNA (Ctr-i). B: The spheroid areas were measured by Image $J$ software. The data are expressed relative to the corresponding value for the cells transfected with control siRNA (Ctri). Significantly different at: ${ }^{* *} p<0.001$.

$p$-value of less than 0.05 was considered to indicate a statistically significant difference.

\section{Results}

Recently, we reported that OIP5-AS1 functions to promote the proliferation of HeLa cervical cancer cells (2). In this study, we first examined whether OIP5-AS1 participates in regulating the proliferation of NSCLC and head and neck squamous cell carcinoma cells. OIP5-AS1 expression was found to be depleted upon transfecting cells with siRNAs (Figure 1, left panels). Silencing OIP5-AS1 resulted in repression of the proliferation of H1299 and A549 NSCLC cells (Figure 1A and B). Silencing OIP-AS1 also repressed the proliferation of $\mathrm{FaDu}$ hypopharyngeal cancer cells, and CAL27 human oral cancer cells (Figure 1C and D). These results suggest that OIP5-AS1 functions to promote the proliferation of these cancer cells.

Cell proliferation is strictly controlled by the cell cycle. Accordingly, we next examined whether OIP5-AS1 is involved in regulating the cell cycle. Cell-cycle analysis demonstrated that silencing OIP-5AS1 significantly increased the proportion of cells in the $\mathrm{G}_{1}$ phase and reduced that of $\mathrm{H} 1299$ and $\mathrm{A} 549$ cells in $\mathrm{S}$ and $\mathrm{G}_{2} / \mathrm{M}$ phases (Figure 2A-C). Silencing OIP5-AS1 also significantly increased the rate of $\mathrm{FaDu}$ and CAL27 cells in the $\mathrm{G}_{1}$ phase (Figure 2D and E), similarly to the case in $\mathrm{H} 1299$ and A549 cells. These results suggested that OIP5-AS1 participates in the $\mathrm{G}_{1}$-phase progression in these cancer cells.

Given that silencing OIP5-AS1 caused $\mathrm{G}_{1}$-phase arrest, we next examined whether OIP5-AS1 participates in regulation of the gene expression of CKIs, which act as brakes on $\mathrm{G}_{1}$-phase progression. RT-qPCR data demonstrated that silencing OIP5AS1 significantly increased the mRNA levels of $p 15$ and $p 19$ in H1299 cells ( $p 16$ was not detected) (Figure 3A) and that of p19 in A549 cells (p15 and p16 were not detected) (Figure 3B). Moreover, silencing OIP5-AS1 significantly increased the mRNA levels of $p 18$ and $p 19$ in FaDu cells ( $p 16$ was not detected) (Figure 3C) and those of $p 15, p 16, p 18$ and $p 19$ in CAL27 cells (Figure 3D). These results suggest that OIP5-AS1 participates in repressing the expression of CKIs such as $p 15$, p16, p18 and $p 19$.

Cancer cells have lost normal cell polarity and obtained the property of anchorage-independent growth through malignant transformation. Accordingly, 3D culture assays are suitable for studying the physiological proliferation of cancer cells. We finally examined whether OIP5-AS1 participates in the regulation of 3D proliferation. Silencing OIP5-AS1 significantly repressed the 3D proliferation of H1299 cells (Figure 4), suggesting that $O I P 5-A S 1$ promotes $3 \mathrm{D}$ proliferation.

\section{Discussion}

Some groups, including us, recently revealed that OIP5-AS1 participates in accelerating the proliferation of several types of cancer cell $(2,3)$. In the current study, we showed that silencing OIP5-AS1 caused cell-cycle arrest at the $\mathrm{G}_{1}$ phase and suppressed the proliferation of H1299 and A549 human NSCLC cells, and FaDu and CAL27 head and neck squamous cell carcinoma cells. We also showed that silencing of OIP5-AS1 increased the expression of CKIs such as p15, p16, p18 and p19, which act as brakes on $\mathrm{G}_{1}$-phase progression in these cells. Taking these findings together, OIP5-AS1 appears to function to accelerate $\mathrm{G}_{1}$-phase progression by repressing $\mathrm{p} 15, \mathrm{p} 16, \mathrm{p} 18$ and p19, resulting in promotion of the proliferation of these cells. The mechanism of action by which OIP5-AS1 represses p15, p16, p18 and p19 is yet to be determined. However, it is known that OIP5-AS1 acts as a sponge for a few dozen miRNAs and competes against them (9). miRNAs participate in the posttranscriptional regulation of gene expression. Accordingly, OIP5-AS1 may repress the expression of p15, p16, p18 and p19 through its interaction with miRNAs. In future work, the involvement of miRNAs in the OIP5-AS1-mediated regulation of CKIs should be examined. 
Recent study revealed that increased expression of OIP5AS1 is observed in many types of human cancer, such as breast, lung, cervical and bladder cancer, glioma, osteosarcoma and hepatoblastoma (3), suggesting that OIP5$A S 1$ acts as an oncogene. However, the physiological relevance of OIP5-AS1 in head and neck squamous cell carcinoma is unclear and requires further study.

In conclusion, we propose that the increased expression of OIP5-AS1 leads to the promotion of $\mathrm{G}_{1}$-phase progression by repressing the expression of CKIs, resulting in tumour promotion.

\section{Conflicts of Interest}

The Authors declare no conflicts of interest directly related to the contents of this article.

\section{Authors' Contributions}

Y.K., N.M., T.W. and R.O. conceptualized and designed the study, performed experiments and analysed data. Y.K. contributed to supervision of the study and wrote the article.

\section{Acknowledgements}

The Authors thank Masahide Kuroki, Kosei Nagayasu and Momoka Ueda for technical support and helpful discussions. This study was supported in part by JSPS KAKENHI Grant Number 17K07184 (to Y.K.), JSPS KAKENHI Grant Number 20K09716 (to T.W.), the Takeda Science Foundation (to Y.K.) and the Naito Foundation (to Y.K.).

\section{References}

1 Ulitsky I, Shkumatava A, Jan $\mathrm{CH}$, Sive $\mathrm{H}$ and Bartel DP: Conserved function of lincRNAs in vertebrate embryonic development despite rapid sequence evolution. Cell 147(7): 15371550, 2011. PMID: 22196729. DOI: 10.1016/j.cell.2011.11.055

2 Naemura M, Kuroki M, Tsunoda T, Arikawa N, Sawata Y, Shirasawa S and Kotake Y: The long noncoding RNA OIP5-AS1 is involved in the regulation of cell proliferation. Anticancer Res 38(1): 77-81, 2018. PMID: 29277759. DOI: 10.21873/anticanres. 12194

3 Li Y, Han X, Feng H and Han J: Long noncoding RNA OIP5AS1 in cancer. Clin Chim Acta 499: 75-80, 2019. PMID: 31476304. DOI: 10.1016/j.cca.2019.08.031

4 Kim J, Abdelmohsen K, Yang X, De S, Grammatikakis I, Noh JH and Gorospe M: LncRNA OIP5-AS1/cyrano sponges RNAbinding protein HuR. Nucleic Acids Res 44(5): 2378-2392, 2016. PMID: 26819413. DOI: 10.1093/nar/gkw017

5 Wang M, Sun X, Yang Y and Jiao W: Long non-coding RNA OIP5-AS1 promotes proliferation of lung cancer cells and leads to poor prognosis by targeting miR-378a-3p. Thorac Cancer 9(8): 939-949, 2018. PMID: 29897167. DOI: 10.1111/17597714.12767
6 Dai J, Xu L, Hu X, Han G, Jiang H, Sun H, Zhu G and Tang X: Long noncoding RNA OIP5-AS1 accelerates CDK14 expression to promote osteosarcoma tumorigenesis via targeting miR-223. Biomed Pharmacother 106: 1441-1447, 2018. PMID: 30119217. DOI: $10.1016 /$ j.biopha.2018.07.109

7 Hu GW, Wu L, Kuang W, Chen Y, Zhu XG, Guo H and Lang HL: Knockdown of linc-OIP5 inhibits proliferation and migration of glioma cells through down-regulation of YAPNOTCH signaling pathway. Gene 610: 24-31, 2017. PMID: 28189759. DOI: $10.1016 /$ j.gene.2017.02.006

8 Zhang Z, Liu F, Yang F and Liu Y: Kockdown of OIP5-AS1 expression inhibits proliferation, metastasis and EMT progress in hepatoblastoma cells through up-regulating miR-186a-5p and down-regulating ZEB1. Biomed Pharmacother 101: 14-23, 2018. PMID: 29475118. DOI: 10.1016/j.biopha.2018.02.026

9 Ghafouri-Fard S, Dashti S, Farsi M, Hussen BM and Taheri M: A review on the role of oncogenic lncRNA OIP5-AS1 in human malignancies. Biomed Pharmacother 137: 111366, 2021. PMID: 33601149. DOI: 10.1016/j.biopha.2021.111366

10 Morgan DO: Principles of CDK regulation. Nature 374(6518): 131-134, 1995. PMID: 7877684. DOI: 10.1038/374131a0

11 Sherr CJ and Roberts JM: CDK inhibitors: positive and negative regulators of G1-phase progression. Genes Dev 13(12): 15011512, 1999. PMID: 10385618. DOI: 10.1101/gad.13.12.1501

12 Matsunaga N, Wakasaki T, Yasumatsu R and Kotake Y: Long noncoding RNA, ANRIL, regulates the proliferation of head and neck squamous cell carcinoma. Anticancer Res 39(8): 40734077, 2019. PMID: 31366490. DOI: 10.21873/anticanres.13564

13 Okamoto H, Matsukawa T, Doi S, Tsunoda T, Sawata Y, Naemura M, Ohnuki K, Shirasawa S and Kotake Y: A novel resveratrol derivative selectively inhibits the proliferation of colorectal cancer cells with KRAS mutation. Mol Cell Biochem 442(1-2): 39-45, 2018. PMID: 28936721. DOI: 10.1007/s11010017-3191-x

14 Kotake $\mathrm{Y}$ and Tsuruda T: Long noncoding RNA ANROC on the INK4 locus functions to suppress cell proliferation. Cancer Genomics Proteomics 17(4): 425-430, 2020. PMID: 32576587. DOI: $10.21873 /$ cgp.20201

15 Naemura M, Murasaki C, Inoue Y, Okamoto H and Kotake Y: Long noncoding RNA ANRIL regulates proliferation of nonsmall cell lung cancer and cervical cancer cells. Anticancer Res 35(10): 5377-5382, 2015. PMID: 26408699.

16 Kotake Y, Goto T, Naemura M, Inoue Y, Okamoto H and Tahara $\mathrm{K}$ : Long noncoding RNA PANDA positively regulates proliferation of osteosarcoma cells. Anticancer Res 37(1): 81-85, 2017. PMID: 28011477. DOI: 10.21873/anticanres.11292

Received May 6, 2021

Revised May 27, 2021

Accepted May 31, 2021 